\title{
InterActua: Modelo de Análise de Ações de Mediação Pedagógica em Ambientes Virtuais de Aprendizagem Baseada em Registros Padronizados
}

\author{
Gustavo Schwarz \\ João Carlos Gluz \\ Programa de Pós-Graduação em Computação Aplicada (PIPCA) - Unisinos - São \\ Leopoldo - RS - Brazil \\ gustavo.sne@gmail.com,jcgluz@unisinos.br
}

\begin{abstract}
Virtual learning environments should not represent only a digital medium for storing and sharing data. They should be useful for students and teachers in the learning mediation. Monitoring activities in these tools is of great importance to make their most efficient use. However, only summarize quantitatively activity records, may not be enough. It is important to assess qualitative issues, identifying features that enable personalized learning. Another problem is that for every existing environment, the records of the activities are stored in a proprietary format. For the present work this problem will be addressed through a computer model based on three fundamental assumptions: a socio-historical approach for education, the use of standards to register activities in an educational environment and the application of Semantic Web technologies to operationalize ontological and dynamic aspects of the model. After the presentation of the main features of this model, this paper concludes by presenting the results of preliminary experiments for evaluating the capabilities of this model.
\end{abstract}

Resumo. Ambientes virtuais de aprendizagem não deve representar somente um meio digital para armazenar e compartilhar dados. Eles devem ser úteis tanto ao aluno quanto ao professor na mediação do aprendizado. $O$ monitoramento das atividades nessas ferramentas é de grande importância para tornar o seu uso mais eficiente. Porém, apenas sumarizar os registros das atividades de forma quantitativa, pode não ser suficiente. É importante avaliar questões qualitativas, identificando características que possibilitem personalizar o ensino. Outro problema encontrado é que para cada ambiente existente, os registros das atividades são armazenados numa estrutura e num formato próprios. Para o presente trabalho essa problemática será abordada através de um modelo computacional alicerçado em três hipóteses fundamentais: a abordagem sócio-histórica da Educação, o uso de padrões para registro de atividades em ambientes educacionais e a aplicação de das tecnologias da Web Semântica para operacionalizar os aspectos ontológicos e dinâmicos do modelo. Após a apresentação das principais características desse modelo, esse trabalho se encerra pela apresentação dos resultados do experimentos preliminares de avaliação das capacidades deste modelo.

\section{Introdução}

A principal inovação das últimas décadas na área de educação foi a criação, implementação e utilização de Ambiente Virtuais de Aprendizagem (AVA), permitindo novas possibilidades e oportunidades educacionais para uma parcela maior da 
população. Os AVA estão cada vez mais presentes no dia-a-dia de professores e alunos, seja no formato exclusivamente a distância ou como uma ferramenta de apoio ao ensino presencial. Apesar do aumento de sua utilização, o uso dessas ferramentas não deve representar somente um meio digital de armazenamento e compartilhamento de dados. Elas também devem ser úteis tanto para o aluno quanto para o professor no processo de mediação do aprendizado. Neste contexto, o monitoramento das atividades nessas ferramentas é de grande importância para tornar o seu uso mais eficiente.

Entretanto, apenas coletar e sumarizar os registros das atividades dos alunos de forma quantitativa - número de acesso, por exemplo - para posteriormente condensálos em relatórios, pode não fornecer informações suficientes para justificar o uso desses ambientes. É importante também avaliar questões qualitativas, identificando características que possibilitem personalizar o ensino. Outro problema encontrado nesses ambientes é que para cada ferramenta existente, os registros das atividades são armazenados numa estrutura e num formato próprios de cada software. Isso, por sua vez, acaba por dificultar a criação de ferramentas padronizadas de análise.

Para o presente trabalho serão abordadas essas duas problemáticas. Primeiramente analisando os mecanismos padronizados de registro das atividades dos participantes em AVA. Em segundo, serão fornecidos mecanismos para a análise desses registros de forma qualitativa utilizando como base a Teoria dos Atos da Fala e a Teoria Sócio-Histórica. Através da pesquisa aqui apresentada objetiva-se fornecer um modelo computacional, denominado InterActua, que possa favorecer o aprendizado através do acompanhamento das atividades de interação social em ambientes virtuais de ensino. Este modelo computacional estará alicerçado em três hipóteses fundamentais alicerçado em três hipóteses fundamentais: a abordagem sócio-histórica da Educação [Vygotsky 1998; Vygotsky 2008], o uso de padrões para registro de atividades em ambientes educacionais e a aplicação de das tecnologias da Web Semântica para operacionalizar os aspectos ontológicos e dinâmicos do modelo. Os objetivos específicos do trabalho são: introduzir os principais conceitos teóricos relevante para a compreensão do processo de pesquisa, analisar o estado da arte das pesquisas relacionadas, apresentar os modelo ontológico e dinâmico de interações educacionais e mostrar os resultados experimentais preliminares obtidos com o modelo. Tais objetivos são alvo das seguintes seções.

\section{Referencial Teórico}

\subsection{Zona de Desenvolvimento Proximal}

A teoria proposta por Vygotsky [1998] aborda o conceito de Zona de Desenvolvimento Proximal (ZDP), que "é a distância entre o nível de desenvolvimento real, que se costuma determinar através da solução independente de problemas e o nível de desenvolvimento potencial, determinado através da solução de problemas sob a orientação de um adulto ou em colaboração com companheiros mais capazes." [Vygotsky 1998; p. 97]

Por sua vez, a ZDP se afirma em contraste ao Nível de Desenvolvimento Real (NDR) (Figura 1(a)), que corresponde aos ciclos de desenvolvimento mental já completados, ou seja, são as capacidades que o indivíduo já possui e que permite-lhe resolver problemas de forma independente. A ZDP define as funções mentais que ainda não amadureceram, mas que estão em processo de maturação, ou seja, o indivíduo consegue resolver um problema mas sob a orientação de um par mais capacitado. Em complemento a isso, [Vicari e Andrade 2006; p. 262] afirmam que "as interações devem 
ocorrer entre pares com níveis cognitivos diversos".

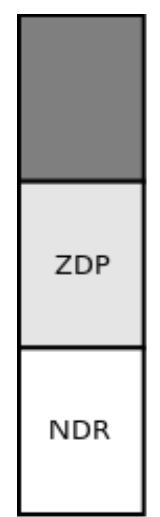

NÃO consegue

solucionar

Precisa de auxílio

para solucionar

Consegue solucionar de forma independente

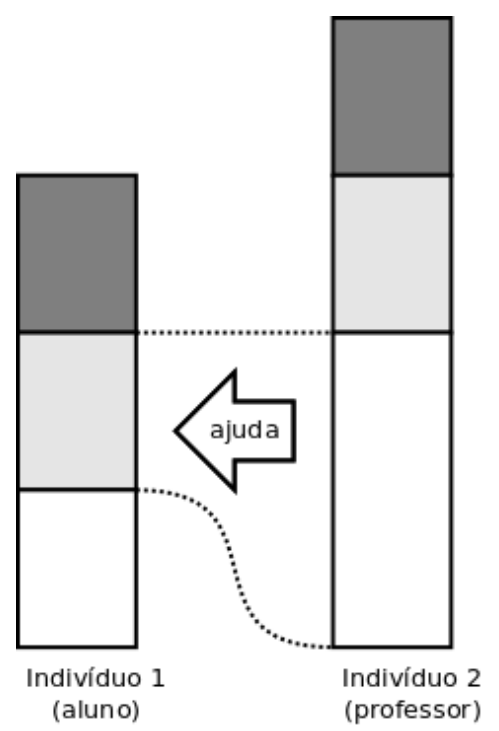

(b) Desenvolvimento da ZDP pela interação social

(a) ZDP e NDR

Figura 1: ZDP, NDR e a interação social (fonte: autor).

Conforme já mencionado a ZDP define as funções mentais que ainda estão em processo de maturação, isso porque a internalização de um conceito acontece de forma gradual, e passa por um processo de mediação. Se a internalização fosse um processo instantâneo, bastaria estar em contato com o objeto do conhecimento para se apropriar de seus conceitos, no entanto as coisas não acontecem de forma tão imediata.

\subsection{Categorias de Mediação}

A mediação é um processo dinâmico onde são utilizadas ferramentas (externas ao sujeito) e signos (internos), o que possibilita ao indivíduo o desenvolvimento dos Processo Psicológicos Superiores (PPS) através da internalização. A ação de mediação é desenvolvida pela interação social entre os indivíduos, os quais, apropriando-se dos instrumentos de mediação, buscam modificar o meio ou o comportamento (seu ou de outra pessoa). Em [Passerino 2005] é possível encontrar uma classificação dessas ações de mediação, sendo elas: controle, autocontrole e autorregulação. O controle é externo ao sujeito e pode ocorrer de forma direta ou indireta. No caso do controle direto ele está divido no seguintes elementos: i) ordens (do tipo imperativo - "Faça assim."); ii) diretivas (ordens mais suaves que geralmente estão no plural - "Vamos clicar aqui."); iii) perguntas diretivas (perguntas com uma ordem implícita - "Podes clicar aqui?"). Já o controle indireto possui o seguintes elementos: i) perguntas perceptivas (perguntas que tenham a resposta no campo perceptivo); ii) perguntas conceituais (perguntas que dependem de uma representação mental para serem respondidas) ; iii) gratificação (uma gratificação, "Muito bom, é isso mesmo!"); iv) renuncia direta ("Agora faça sozinho."); v) confirmação passiva (o sujeito mais experiente responde às questões dirigidas a ele pelo sujeito menos experiente); vi) afastamento físico (o sujeito mais experiente deixa o aluno só, ficando apenas na observação e intervindo caso necessário). 


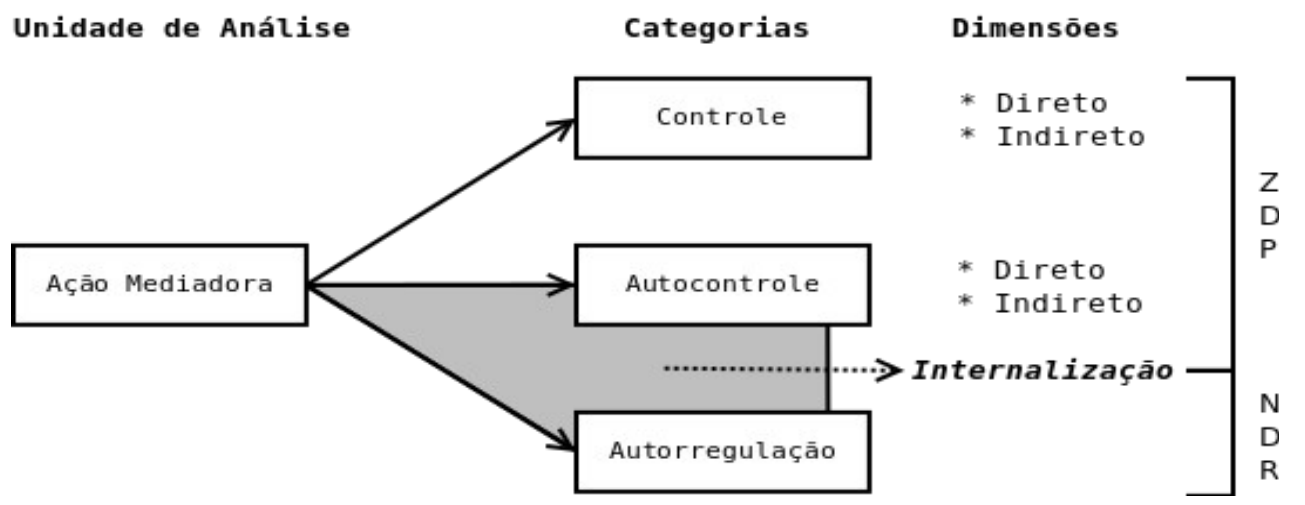

Figura 2. Categorias de Regulação das Ações Mediadoras (fonte: [Passerino 2005])

O autocontrole representa uma ação realizada pelo sujeito obedecendo a um tutor internalizado. $\mathrm{O}$ autocontrole direto está dividido em: i) imitação direta (processo de mediação idêntico ao realizado pelo mediador); modelo (baseado na imitação, no entanto novos elementos são introduzidos); fala dirigida (antecipação verbal das ações que serão desenvolvidas dirigidas ao mediador esperando confirmação). O autocontrole indireto corresponde a fala que o sujeito dirige a si mesmo. A autorregulação é o plano de ação que o sujeito cria e que se converte em seu próprio tutor.

\subsection{Teoria dos Atos da Fala}

No mediação para transmitir uma experiência de forma racional e intencional para outro, é necessário um sistema mediador, cujo protótipo é a fala humana, tendo essa o papel de intercâmbio social. [Vygotsky 2008]. Para realizar uma análise da comunicação, o primeiro passo é a observação da fala em si, analisando seus elementos ou estágios componentes. Austin [1990] caracterizou o ato de enunciação de uma frase, ou oração de um falante a um ouvinte, como um Ato da Fala que pode ser dividido em três tipos (ou níveis) distintos de Atos da Fala: (a) os atos locucionários, que corresponde a enunciação efetiva dos fonemas, sílabas e palavras do falante; (b) os atos ilocucionários, que compreende o significado que o falante procura associar ao seu ato locucionário e que está tentando transmitir ao ouvinte; (c) os atos perlocucionários: associado aos efeitos ocasionados ao ouvinte em função da enunciação do ato da fala em si. Searle [2002] faz um revisão dos atos ilocucionários, tomando como base as teorias de Austin e propondo novo critérios de classificação, resumidos na Tabela 1.

Tabela 1: Categorias Básicas dos Atos llocucionários (fonte: [Searle 2002]).

\begin{tabular}{|l|l|}
\hline Força/Tipo & Descrição \\
\hline Assertivo & $\begin{array}{l}\text { O propósito dos membros da classe assertiva é o de comprometer o falante com a } \\
\text { verdade da proposição expressa. }\end{array}$ \\
\hline Diretivo & $\begin{array}{l}\text { Seu propósito ilocucionário consiste no fato de que são tentativas do falante de } \\
\text { levar o ouvinte a fazer algo. }\end{array}$ \\
\hline Compromissivo & $\begin{array}{l}\text { Os compromissivos são os atos ilocucionários cujo propósito é comprometer o } \\
\text { falante com alguma linha futura de ação. }\end{array}$ \\
\hline Expressivo & $\begin{array}{l}\text { O propósito dessa classe é o de expressar um estado psicológico. Exemplos de } \\
\text { verbos desse grupo são: thank (agradecer), congratulate (congratular), apologize } \\
\text { (desculpar-se), welcome (dar boas-vindas). }\end{array}$ \\
\hline Declarativo & Indica a realização bem-sucedida entre o conteúdo proposicional e a realidade. \\
\hline
\end{tabular}


Cabe ressaltar que a comunicação não ocorre somente pela transmissão de signos (a palavra ou som), pois a verdadeira comunicação requer significado - isto é generalização - tanto quanto signos [Vygotsky 2008]. Nos estudos de Searle [2002] o significado começa a ser evidenciado através da força ou do tipo do atos ilocucionários, dependendo da categorização assumida em ato assertivo (e. g. concluir, deduzir), diretivo (e. g. convidar, pedir), compromisso (e. g. prometer), expressivos (e. g. agradecer, desculpar), declarativo (declarar algo, e. g. "Você está demitido").

\section{Trabalhos Relacionados}

Nas pesquisas por trabalhos relacionados objetivou-se identificar os trabalhos que utilizassem da teoria sócio-histórica e sua relação ao processo de mediação nos ambientes virtuais de ensino. Em [Mazzoni e Gaffuri 2009] é utilizada a abordagem sócio-histórica como base teórica do trabalho, na tentativa de identificar as ações dos alunos nos ambientes educacionais web. Para a identificação das ações individuais, os autores realizaram uma análise de arquivos de $\log$ e de bases de dados. Para a análise das ações coletivas, são utilizadas técnicas de Análise de Rede Social sobre as trocas de mensagens entre os participantes das ferramentas colaborativas (chat, fórum, etc). Apesar de ser possível identificar a rede de relacionamentos dos participantes e as suas ações individuais, não há evidências que indiquem algum processo de mediação no ambiente analisado. No trabalho de [Severo et al. 2009] é elaborada uma ontologia para as categorias de mediação. No entanto, não foi possível identificar onde a ontologia estava sendo utilizada. Em [Rios et al. 2008] é realizada uma análise levando em consideração fatores importantes como a qualidade das interações de aluno-aluno e professor-aluno em AVA. A coleta de dados foi realizada através de questionários e o dados foram tabulados manualmente. Assim, aplicar essa mesma metodologia em um cenário real pode tornar-se bastante oneroso para professor. No trabalho [Koch et al. 2009] é realizado um estudo para identificar as categorias de mediação em ferramentas de e-mail e portfólio, sendo apresentadas evidências da utilidade destas ferramentas no processo de mediação. No estudo [Machado, 2009] são identificados pontos importantes da mediação pedagógica como a avaliação das interações nos fóruns, o nível de participação, quais atividades motivaram os alunos, entre outras. No entanto estes dados foram coletados e sumarizados de forma manual, o que poderia levar a uma sobrecarga do professor. Em [Severo et al. 2011] é elaborado um sistema para identificação de categorias de mediação no ambiente Moodle, baseado em mineração de textos sobre os registros das ferramentas de chat e fórum.

Todos os trabalhos possuem em comum a abordagem sócio-histórica como base epistemológica de suas pesquisas. Entretanto, existem diferenciais importantes do modelo InterActua, identificados a seguir. Com exceção da ontologia de [Severo et al. 2009], nestes trabalhos são realizadas análises do ensino em ferramentas de comunicação como fórum, chat e e-mail. As demais ferramentas, como as de tarefas, atividades, não são analisadas frente as possibilidades que podem fornecer ao processo de ensino-aprendizagem. Desta forma, no presente trabalho pretende-se abordar outras ferramentas. A maioria das ferramentas relatadas nos trabalhos relacionados utiliza mecanismos manuais para a coleta e sumarização das informações. Segundo constata [Medeiros e Gomes 2012], monitorar e analisar as informações é um fator importante para o instrutor, mas, dependendo do número de alunos, a falta de mecanismos automatizados pode tornar essa tarefa tediosa, quase impossível. Outro ponto considerado na análise é a questão de interoperabilidade, não sendo possível observar este elemento nos trabalhos citados. O padrão escolhido, Tincan [ADL 2013], visa 
abordar essa questão, sendo seu foco voltado para os metadados das experiências de aprendizagem

\section{Modelo Ontológico de Mediação baseado em Registros de Atividades}

$\mathrm{Na}$ teoria sócio-histórica de Vygotsky o aprendizado é tido como prática social. Os instrumentos e os signos, juntamente com as interações dos indivíduos com o ambiente e com a sociedade, representam elementos importantes no processo de desenvolvimento humano. Estes elementos não estão presentes somente em ambientes presenciais, mas também nos ambientes virtuais de ensino utilizados atualmente. Para o presente trabalho procurou-se representar computacionalmente esses elementos em formatos padronizados, assim como utilizar um modelo semântico para essa representação. $\mathrm{O}$ modelo ontológico resultante é formado por duas ontologias apresentadas a seguir.

\subsection{Ontologia de Metadados Tincan}

A ontologia que descreve os registros de interação foi criada com base no padrão Tincan [ADL 2013]. Nas pesquisas não foi possível identificar uma ontologia previamente definida, dessa forma optou-se pela elaboração de uma para o presente trabalho. Os três principais elementos obrigatórios de cada registro Tincan são o sujeito, o verbo e o objeto. Na criação da ontologia Tincan, primeiramente foram definidas as classes e as relações conforme a estruturação da especificação oficial dest padrão [ADL 2013]. A Figura 3 mostra como está organizada a hierarquia de classes dessa ontologia.
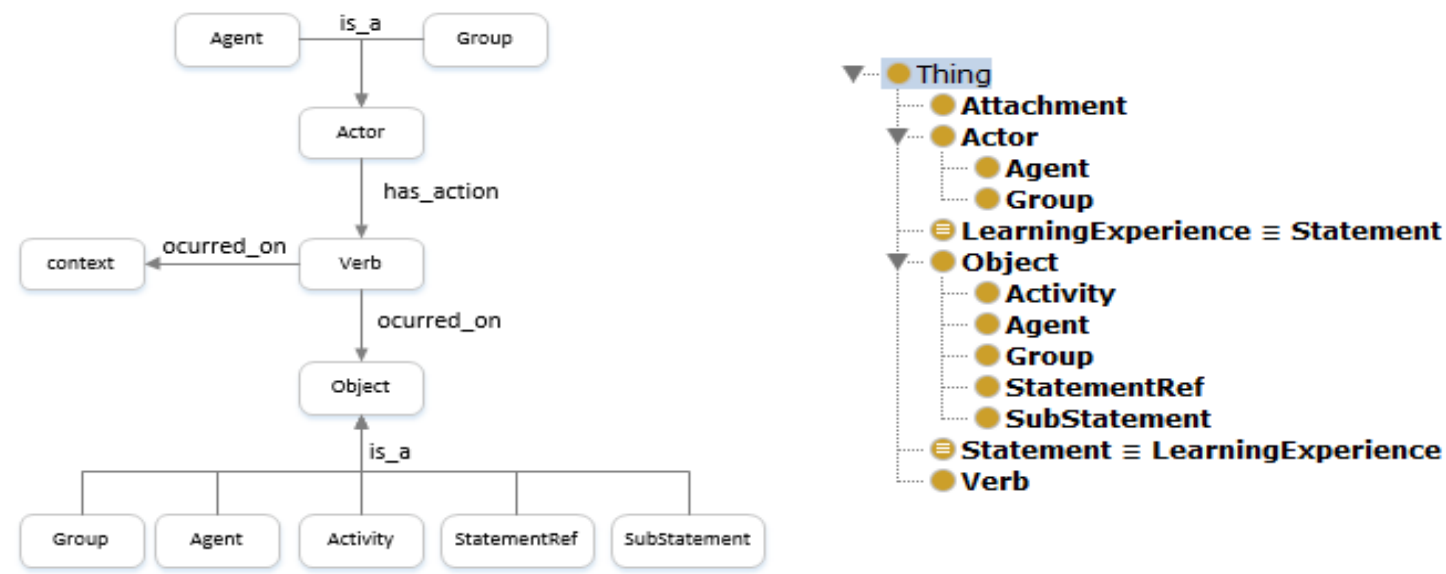

Figura 3. llustração Simplificada da Ontologia Tincan (fonte: autor)

\subsection{Ontologia Conceitual de Ações de Mediação}

A ontologia de mediação define um vocabulário OWL para as ações de mediação. Tem como base o trabalho de Severo et al. [2009], generalizando a estrutura conceitual definida neste trabalho pela inclusão de uma categorização das ações de mediação integrada a classificação das forças ou tipos de atos da fala.

A ontologia está divida da seguinte maneira: (a) Ações de Mediação (AcaoMediacao): nova estrutura conceitual para as ções de mediação que representa as ações comunicativas, levando em conta a classificação dos atos da fala. Também representa ações físicas (um afastamento físico, por exemplo); (b) Categorias Cognitivas (CategoriaCognitiva): nova classificação que compreende os elementos cognitivos abordados na teoria sócio-histórica, que são: a ZDP, o NDR e os Processos 
Psicológicos Superiores (PPS); (c) Categorias de Mediação (CategoriaMediacao): são as mesmas classes e subclasses das categorias de mediação apresentadas em Severo et al. [2009]: Controle, Autocontrole e Autorregulação. (d) Indivíduos (Sujeitos): representam os sujeitos no processo de mediação, sendo eles: o par mais capaz (professor ou mediador) e o par menos capaz (aluno).

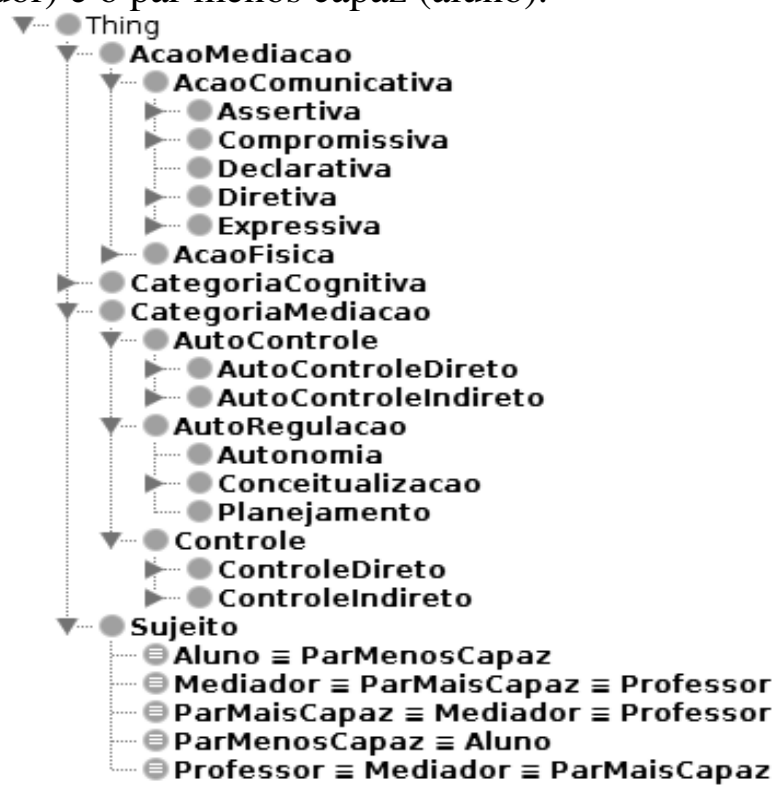

Figura 4. Ontologia de Ações de Mediação (fonte: autor)

\section{Modelo Dinâmico de Classificação das Interações}

A Figura 5 mostra a visão geral do modelo dinâmico de acompanhamento das interações. O Moodle, a base de de registros de log deste AVA e o software de marcação sintática de textos da Língua Portuguesa Aelius são elementos externos ao modelo, não tendo sido desenvolvidos no contexto deste trabalho. A escolha do Moodle como AVA a ser integrado ao modelo é devida ao seu amplo uso em instituições de ensino [Penteado et al. 2014]. Aelius [Alencar 2013] é um projeto gratuito e de código aberto desenvolvido em Python que permite a marcação sintática de textos em português brasileiro

O processo Conversor Log para Tincan é responsável pela conversão dos registros contidos no log do AVA para o formato Tincan. A versão atual suporta a conversão dos registros de log das ferramentas Atividade, Curso, Fórum, e Avaliação, além da postagem conteúdos Página e Recurso do Moodle. A extração das informações referentes ao sujeito e ao objeto de um registro Tincan podem ser feitas com base nas informações de usuário e de recurso utilizado contidas nos registros de $\log$ do Moodle. Para a extração do tipo de verbo de um registro Tincan, foi necessário utilizar em alguns casos o marcador sintático Aelius [Alencar 2013] de forma identificar corretamente o tipo de verbo vinculado ao registro. O processo Conversor Tincan para RDF, transforma as triplas Sujeito-Verbo-Objeto do formato Tincan em triplas RDF Predicado-Sujeito-Objeto. Os elementos Sujeito e Objeto são, quando necessário, convertidos para o formato URI usado em RDF. Os identificadores de verbos são convertidos em identificadores de predicados com o mesmo prefixo da ontologia Tincan, sendo categorizados como subclasses da classe Verb. Também é feita uma classificação do sujeito e do objeto, respectivamente, nas subclasses da classe Actor e Object. 


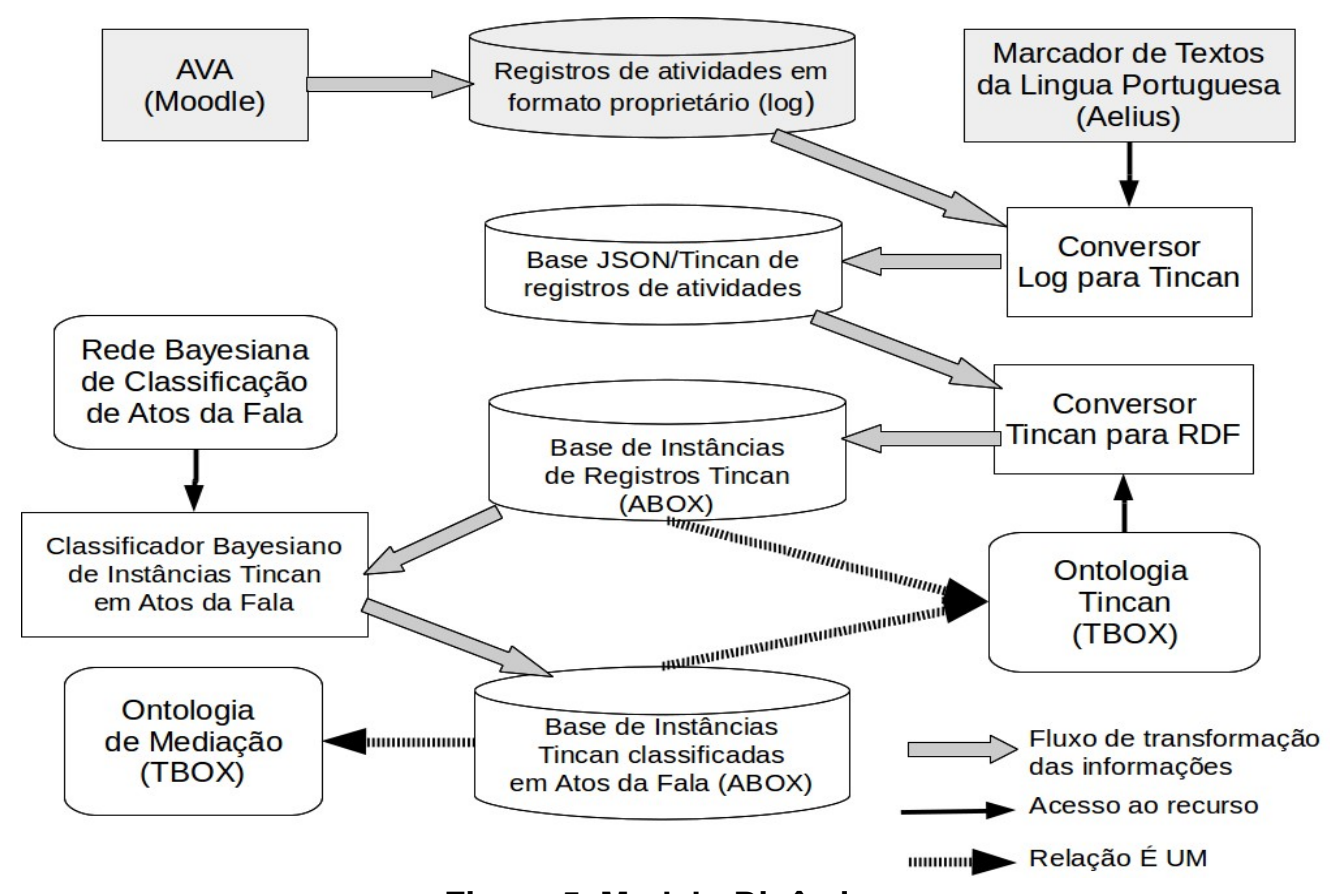

Figura 5. Modelo Dinâmico

Para a classificação dos registros de atividades Tincan já representados na forma de instâncias da ontologia de metadados Tincan foi criada uma Rede Bayesiana de classificação baseada no modelo Naive-Bayes, que, a partir das evidências fornecidas pelo registro de atividades, identifica a subclasse da classe AcaoComunicativa da ontologia de Ações de Mediação que este registro pode pertencer. A topologia da Rede Bayesiana de classificação foi criada manualmente, enquanto que os parâmetros quantitativos foram baseados na análise estatística de um conjunto de registros de atividades Tincan, previamente coletado e classificado manualmente. $\mathrm{O}$ resultado final do modelo dinâmico é uma base de instâncias de registros Tincan, onde as diversas atividades dos alunos e professores no sistema estão classificadas de acordo com atos comunicativos assertivos, compromissivos, declarativos ou diretivos [Searle 2002].

\section{Experimentos}

A coleta dos dados no ambiente Moodle foi realizado em uma turma de Metodologia de Pesquisa da Universidade do Vale do Rio dos Sinos (UNISINOS) que ocorreu no primeiro semestre de 2014. Na época a versão estável do Moodle era a 2.6, portanto essa versão foi usada como base para os experimentos. Foram coletados um total de 4.567 registros de $\log$. Desses foram excluídos 1.172 registros administrativos, resultando 3.395 registros de log das atividades de Fórum, Curso, Avaliação e postagens de páginas e recursos obtidas durante aproximadamente 3 meses de duração da disciplina. Todos estes registros foram então convertidos, pelo processo Conversor Log para Tincan para registros no padrão Tincan em formato JSON, atingindo a taxa de $100 \%$ de conversão. Após, todos os registros de atividade Tincan foram convertidos para o formato RDF, também atingindo $100 \%$ de conversão.

Posteriormente, essa base de registros Tincan em formato RDF foi analisada manualmente, sendo atribuída uma classe de AcaoComunicativa para cada um deles. Tal base classificada foi utilizada para a modelagem dos parâmetros quantitativos da Rede Bayesiana de Classificação de Atos da Fala. O processo Classificador de 
Instâncias Tincan de Atos da Fala foi validado através da seleção aleatória de uma amostra de 150 registros da base Tincan em formato RDF. O classificador foi aplicado aos registros dessa amostra. Os resultados das previsões do classificador foram comparados a classificação manual original, resultando nas matrizes de confusão apresentadas na Tabela 1, que foram construídas para cada uma das classificações de atos da fala possíveis de serem feitas pelo classificador.

Tabela 2 - Matrizes de confusão do classificador de atos da fala

\begin{tabular}{|c|c|c|c|c|c|c|c|c|}
\cline { 2 - 8 } \multicolumn{1}{c|}{} & \multicolumn{2}{c|}{ Atos assertivos } & \multicolumn{2}{c|}{ Atos diretivos } & \multicolumn{2}{c|}{ Atos declarativos } & \multicolumn{2}{c|}{ Atos compromissivos } \\
\cline { 2 - 9 } \multicolumn{1}{c|}{} & \multicolumn{2}{c|}{ Previsto } & \multicolumn{2}{c|}{ Previsto } & \multicolumn{2}{c|}{ Previsto } & \multicolumn{2}{c|}{ Previsto } \\
\hline Real & Positivo & Negativo & Positivo & Negativo & Positivo & Negativo & Positivo & Negativo \\
\hline Positivo & $13.3 \%$ & $2.0 \%$ & $7.3 \%$ & $0.7 \%$ & $14.7 \%$ & $8.7 \%$ & $21.3 \%$ & $6.0 \%$ \\
\hline Negativo & $7.3 \%$ & $77.3 \%$ & $6.0 \%$ & $86.0 \%$ & $17.3 \%$ & $59.3 \%$ & $12.7 \%$ & $60.0 \%$ \\
\hline
\end{tabular}

Essas matrizes permitem inferir os coeficientes de erro, acurácia, precisão e sensibilidade (recall) para cada uma das possíveis classificação de atos da fala, apresentadas na tabela 3 .

Tabela 3 - Coeficientes de erro, acurácia, precisão e sensibilidade

\begin{tabular}{|c|c|c|c|c|}
\cline { 2 - 5 } \multicolumn{1}{c|}{} & Atos assertivos & Atos diretivos & Atos declarativos & Atos compromissivos \\
\hline Erro & 0.093 & 0.067 & 0.260 & 0.187 \\
\hline Acurácia & 0.907 & 0.933 & 0.740 & 0.813 \\
\hline Precisão & 0.645 & 0.550 & 0.458 & 0.627 \\
\hline Sensibilidade & 0.870 & 0.917 & 0.629 & 0.780 \\
\hline
\end{tabular}

Os altos coeficientes de acurácia e baixos coeficientes de erro, que indicam, respectivamente, o percentual de total de acertos e total de erros sobre o total de registros analisados, fornecem boas evidências que o classificador pode ser utilizado para fins práticos. Entretanto, os coeficientes de precisão, que indicam o percentual de verdadeiros positivos sobre o total de verdadeiros positivos e falsos positivos, possuem valores relativamente baixos, indicando possíveis melhorias.

\section{Considerações Finais}

Este artigo apresenta o modelo computacional InterActua que é capaz de classificar os registros de atividades alunos e professores em um AVA em atos comunicativos baseados nos tipos de Atos da Fala propostos por Searle [2002]. Um resultado secundário, mas não menos importante do modelo, é sua capacidade de conversão dos registros de atividade proprietários em registros padronizados no formato Tincan, seguida da transformação destes registros em bases de informações (triplas RDF) que podem ser tratadas pela tecnologia de ontologias OWL.

O modelo InterActua é, entretanto, apenas um passo inicial do processo de análise e identificação das categorias de regulação da mediação, como controle e autocontrole, que podem ser evidenciadas em um processo de ensino e aprendizagem, segundo a abordagem sócio-histórica. Apesar disso, as evidências iniciais de efetividade dos processos de conversão e classificação de registros educacionais alcançados pelo InterActua, são indícios da viabilidade deste método de análise de atos de comunicação. A organização ontológica do modelo InterActua, que já prevê a possibilidade de interrelacionar classes ou tipos de atos de comunicação com ações de mediação, também indica um caminho subsequente de pesquisa, para operacionalizar a detecção e 
identificação das categorias de regulação da mediação em registros educacionais.

\section{Referências Bibliográficas}

ADL (2013) Advanced Distributed Learning - Experience API. http://github.com/adlnet/xAPI-Spec

Alencar, L. F. (2013) Aelius User's Manual. http//aelius.sourceforge.net/manual.html

Austin, J. L. (1990) Quando dizer é fazer: Palavras e Ação, Artes Médicas, Porto Alegre.

Koch, S. H. d. S., Maciel, M. e Passerino, L. M. (2009) "The mediation in distance learning: possibilities of mapping the signs", In Anais do IFIP World Conference on Computers in Education.

Mazzoni, E. e Gaffuri, P. (2009) "Monitoring activity in e-learning: A quantitative model based on web tracking and Social Network Analysis", In: Monitoring and Assessment in Online Collaborative Environments: Emergent Computational Technologies for E-Learning Support, IGI Global, p.111-129.

Medeiros, F. P. A. e Gomes, A. S. (2012) "Monitoramento da Experiência do Usuário em Ambientes Colaborativos Virtuais de Aprendizagem: Um Mapeamento Sistemático", In Anais do Anais do XXIII Simpósio Brasileiro de Informática na Educação (SBIE 2012).

Passerino, L. M. (2005) Pessoas com autismo em ambientes digitais de aprendizagem: estudo dos processos de interação social e mediação (Tese de Doutorado), Universidade Federal do Rio Grande do Sul (UFRGS).

Penteado, F., Gluz, J. C. e Galafassi, C. (2014) "Análise Crítica das Pesquisas Recentes sobre as Tecnologias de Objetos de Aprendizagem e Ambientes Virtuais de Aprendizagem", Revista Brasileira de Informática na Educação (RBIE), 21, p.41-52.

Rios, J., Pimentel, R. e Silva, B. D. (2008) "Mediação pedagógica online: análise transversal de ferramentas de interação", In Anais do COLÓQUIO LUSOBRASILEIRO SOBRE QUESTÕES CURRICULARES, 4, Universidade de Santa Catarina/Brasil, Florianópolis, Brasil.

Searle, J. R. (2002) Expressão e Significado, Martins Fontes.

Severo, C. E. P., Passerino, L. M., Gluz, J. C. e Raminelli, A. (2011) "Mediação pedagógica em ambientes virtuais de ensino-aprendizagem através de agentes de mineração de dados educacionais", Informática na Educação (IETP), 14(3), p.63-82.

Severo, C. E. P., Passerino, L. M., Koch, S. H. S., Maciel, M. C. P. e Gluz, J. C. (2009) "Uma ontologia para categorias de mediação segundo uma abordagem epistemológica baseada na interação social", RENOTE. Revista Novas Tecnologias na Educação, 7(3), p.1-11.

Vicari, R. M. e Andrade, A. F. (2006) Construindo um ambiente de aprendizagem a distância inspirado na concepção sociointeracionista de Vygotsky, Teorias, práticas, legislação e formação corporativa. Edições Loyola. p. 257-274, Educação Online.

Vygotsky, L. S. (1998) Formação Social da Mente, Martins Fontes, São Paulo.

Vygotsky, L. S. (2008) Pensamento e Linguagem, Martins Fontes, São Paulo. 